\title{
On totally umbilic submanifolds of semi-Riemannian manifolds
}

\author{
Volker Perlick \\ Institut für Theoretische Physik, Sekr. PN 7-1 \\ TU Berlin, 10623 Berlin, Germany \\ Email: vper0433@itp.physik.tu-berlin.de
}

\begin{abstract}
The notion of being totally umbilic is considered for non-degenerate and degenerate submanifolds of semi-Riemanian manifolds. After some remarks on the general case, timelike and lightlike totally umbilic submanifolds of Lorentzian manifolds are discussed, along with their physical interpretation in view of general relativity. In particular, the mathematical notion of totally umbilic submanifolds is linked to the notions of photon surfaces and of null strings which have been used in the physics literature.
\end{abstract}

\section{Introduction}

In a Riemannian manifold, a submanifold is said to have an umbilic point at $p$ if, at this point $p$, the second fundamental form is a multiple of the first fundamental form. A submanifold is called totally umbilic if all of its points are umbilic. In $n$-dimensional Euclidean space, a $k$-dimensional complete connected submanifold with $2 \leq k \leq n-1$ is totally umbilic if and only if it is a $k$-sphere or a $k$-plane.

If we generalize from Riemannian to semi-Riemannian manifolds (i.e., if the metric of the ambient space need not be positive-definite), a submanifold may be degenerate (i.e., the metric may induce a degenerate tensor field on this submanifold). In this case the standard text-book definition of the second fundamental form (or shape tensor) does not make sense; so many authors restrict the definition of totally umbilic submanifolds to the non-degenerate case, see, e.g., O'Neill [1. However, there is a fairly obvious way in which the definition of the second fundamental form can be generalized to include degenerate submanifolds. This is indicated in Exercise 9 on p.125 in O'Neill's book [1] but not used in the main part. The geometry of degenerate submanifolds of semi-Riemannian manifolds is discussed in detail in the books by Kupeli [2] and by Duggal and Bejancu [3]

In this article I use the generalized definition of the second fundamental form, as suggested in the above-mentioned exercise of O'Neill's book, and discuss, thereupon, some general properties of totally umbilic submanifolds which may or may not be degenerate. In the second part of the article I specialize to the case that the ambient space has Lorentzian signature and give several characterizations of totally umbilic submanifolds that are timelike or lightlike. The interpretation of these results in view of general relativity is also discussed. It is one of the main purposes of this article to link up the mathematical literature on totally umbilic submanifolds with some notions used in the physical literature; these are, in particular the notions of photon surfaces (introduced 
by Claudel, Virbhadra and Ellis [4] and also discussed by Foertsch, Hasse and Perlick [5]) and of null strings (introduced by Schild [12]).

\section{Definition and general properties of totally umbilic sub- manifolds in semi-Riemannian manifolds}

Let $(M, g)$ be a semi-Riemannian manifold and $\nabla$ the Levi-Civita connection of $g$. On every (immersed) submanifold $\tilde{M}$ of $M, g$ induces a second-rank tensor field $\tilde{g}$. If $\tilde{g}$ has a non-trivial kernel, the submanifold is called degenerate; otherwise it is called non-degenerate.

In the non-degenerate case $(\tilde{M}, \tilde{g})$ is a semi-Riemannian manifold in its own right; in particular, it defines a Levi-Civita connection on $\tilde{M}$. In the degenerate case, the ambient space induces, in general, no distinguished connection on $\tilde{M}$

Moreover, in the non-degenerate case at each point $p$ in $\tilde{M}$ the tangent space splits orthogonally,

$$
T_{p} M=T_{p} \tilde{M}+T_{p}^{\perp} \tilde{M}
$$

where $T_{p}^{\perp} \tilde{M}$ denotes the set of all vectors in $T_{p} M$ that are perpendicular to $T_{p} \tilde{M}$ with respect to $g$. In the degenerate case, (11) does not hold because $T_{p} \tilde{M}$ and $T_{p}^{\perp} \tilde{M}$ have a non-trivial intersection and do not span the whole tangent space $T_{p} M$. In other words, a vector in $T_{p} M$ cannot be decomposed uniquely into a component tangent to $\tilde{M}$ and a component perpendicular to $\tilde{M}$. This is the reason why the standard text-book definition of the second fundamental form, which makes use of this decomposition, does not work. However, this problem can be easily circumvented by using the quotient space $T_{p} M / T_{p} \tilde{M}$ instead of $T_{p}^{\perp} \tilde{M}$. We denote the elements of this quotient space by square brackets, i.e., we write

$$
\left[Z_{p}\right]=\left\{Z_{p}+Y_{p} \mid Y_{p} \in T_{p} \tilde{M}\right\}
$$

for $Z_{p} \in T_{p} M$. If $Z$ is a vector field along $\tilde{M}$, we denote by $[Z]$ the map that assigns to each point $p \in T_{p} \tilde{M}$ the equivalence class $\left[Z_{p}\right]$, where $Z_{p}$ is the value of $Z$ at $p$. Using this notation, we define the second fundamental form $\Pi$ of $\tilde{M}$ by the equation

$$
\Pi(X, Y)=\left[\nabla_{X} Y\right]
$$

where $X$ and $Y$ are vector fields tangent to $\tilde{M}$. As $\nabla$ is torsion free, $\nabla_{X} Y-\nabla_{Y} X=[X, Y]$, and as with $X$ and $Y$ also the Lie bracket $[X, Y]$ must be tangent to $\tilde{M}$, it is clear that $\Pi$ is symmetric, $\Pi(X, Y)=\Pi(Y, X)$. This symmetry property implies that $\Pi$ is tensorial with respect to both arguments, because it is obviously tensorial with respect to the first one. This reasoning is quite analogous as for the standard text-book definition of the second fundamental form; the only difference to this standard definition lies in the fact that at each point $p \in \tilde{M}$ now $\Pi(X, Y)$ takes values in $T_{p} M / T_{p} \tilde{M}$ rather than in $T_{p}^{\perp} \tilde{M}$. For non-degenerate submanifolds, these two spaces can of course be identified in a natural fashion.

With $\Pi$ given by (3), we can now define the notion of being totally umbilic for submanifolds that may be degenerate or non-degenerate.

Definition 1. A submanifold $\tilde{M}$ of a semi-Riemannian manifold is called totally umbilic if there is a vector field $N$ along $\tilde{M}$ such that

$$
\Pi(X, Y)=[g(X, Y) N]
$$


for all vector fields $X$ and $Y$ tangent to $\tilde{M}$. A totally umbilic submanifold with $[N]=[0]$ is called totally geodesic.

Note that the property of being totally umbilic is invariant under conformal changes of $g$ whereas the property of being totally geodesic is not. Also note that for a totally umbilic submanifold the equivalence class $[N]$ is unique but the vector field $N$ is not. In the non-degenerate case we can make $N$ unique by requiring that it be perpendicular to $\tilde{M}$. In the totally geodesic case, we can of course choose $N=0$. For degenerate totally umbilic submanifolds that are not totally geodesic, however, there is no distinguished choice for the vector field $N$.

Once $N$ has been chosen, the equation

$$
\tilde{\nabla}_{X} Y=\nabla_{X} Y-g(X, Y) N
$$

defines a torsion-free connection $\tilde{\nabla}$ on $\tilde{M}$. For non-degenerate totally geodesic submanifolds, $\tilde{\nabla}$ with the choice $N=0$ coincides with the Levi-Civita connection of $(\tilde{M}, \tilde{g})$.

We now prove a proposition which is a simple consequence of the existence of the connection (5). In this proposition we use the following terminology. (This terminology comes from general relativity and is, actually, motivated only in the case that the metric of the ambient space has Lorentzian signature.) We call a vector field $X$ on $M$ lightlike if $g(X, X)=0$; a curve is called lightlike if it is the integral curve of a lightlike vector field.

Proposition 1. (a) Let $\tilde{M}$ be a totally umbilic submanifold of $M$. Then a lightlike $\nabla$-geodesic that starts tangential to $\tilde{M}$ remains within $\tilde{M}$ (for some parameter interval around the starting point).

(b) $\tilde{M}$ is totally geodesic if and only if every $\nabla$-geodesic that starts tangential to $\tilde{M}$ remains within $\tilde{M}$ (for some parameter interval around the starting point).

Proof. From (5) we read that for lightlike vector fields on $\tilde{M}$ the equation $\tilde{\nabla}_{X} X=0$ is equivalent to $\nabla_{X} X=0$. In other words, the $\nabla$-geodesics with lightlike initial vectors tangent to $\tilde{M}$ are $\tilde{\nabla}$ geodesics and thus remain within $\tilde{M}$. This proves (a). In the totally geodesic case we may choose $N=0$, so the same argument works for non-lightlike initial vectors as well. This proves (b).

Part (b) is, of course, the true justification for the name 'totally geodesic'.

The following characterization is often useful.

Proposition 2. (a) $\tilde{M}$ is totally umbilic if and only if all vector fields $X$ and $Y$ which are tangent to $\tilde{M}$ with $g(X, Y)=0$ satisfy $\Pi(X, Y)=[0]$.

(b) $\tilde{M}$ is totally geodesic if and only if all vector fields $X$ and $Y$ which are tangent to $\tilde{M}$ satisfy $\Pi(X, Y)=[0]$.

Proof. Claim (b) and the 'only if' part of claim (a) are obvious from Definition 1 To prove the 'if' part of claim (a), we choose basis vector fields $E_{1}, \ldots, E_{l}, L_{1}, \ldots, L_{m}$ on $\tilde{M}$ such that the $E_{i}$ are pseudo-orthonormal and $g\left(L_{\mu}, L_{\nu}\right)=g\left(L_{\mu}, E_{i}\right)=0$ for all $\mu, \nu=1, \ldots, m$ and all $i=1, \ldots, l$. For each $i$, we define a vector field $N_{i}$ along $\tilde{M}$ by $\nabla_{E_{i}} E_{i}=g\left(E_{i}, E_{i}\right) N_{i}$. Considering for $X$ and $Y$ all linear combinations of $E_{i}$ and $E_{j}$ that are orthogonal to each other, our hypothesis implies that $\left[N_{i}\right]=\left[N_{j}\right]=:[N]$ for all $i, j=1, \ldots, l$. With this information at hand, we consider for $X$ and $Y$ arbitray vector fields tangent to $\tilde{M}$, i.e., linear combinations of all $E_{i}$ and $L_{\mu}$; then our hypopthesis implies that $\Pi(X, Y)$ is, indeed, of the form (4). 
It follows directly from Definition 1 that a non-degenerate one-dimensional submanifold is always totally umbilic and that a degenerate one-dimensional submanifold is totally umbilic if and only if it is the image of a geodesic. For this reason the notion of totally umbilic submanifolds is non-trivial only for $1<\operatorname{dim}(\tilde{M})<\operatorname{dim}(M)$. In an arbitrary semi-Riemannian manifold, the existence of non-trivial totally umbilic submanifolds is not guaranteed. For the case that the ambient space is Riemannian, existence criteria for totally umbilic foliations in terms of curvature conditions have been given by Walschap 9. Generalizations to the semi-Riemannian case have, apparently, not been worked out so far. Here are two simple examples of semi-Riemannian manifolds that do admit non-trivial totally umbilic submanifolds.

Example 1. O'Neill 1], p.117, considers the case where $(M, g)$ is pseudo-Euclidean, i.e. $\mathbb{R}^{n}$ with a constant metric of arbitrary signature. He shows that for $n \geq 3$ a complete connected nondegenerate hypersurface is totally umbilic if and only if it is either a hyperplane or a hyperquadric. Every connected non-degenerate totally umbilic submanifold $\tilde{M}$ with $2 \leq \operatorname{dim}(\tilde{M}) \leq \operatorname{dim}(M)-2$ is a hypersurface in some pseudo-Euclidean subspace of dimension $\operatorname{dim}(\tilde{M})+1$; this was proven by Ahn, Kim, and Kim [10] (cf. Hong [1] for the Lorentzian case). Thus, with the O'Neill result we know all non-degenerate totally umbilic submanifolds of pseudo-Euclidean space.

Example 2. Consider the case that $(M, g)$ is (locally) a twisted product. By definition, this means that $M$ admits coordinates $(u, v)=\left(u^{1}, \ldots, u^{m}, v^{1}, \ldots, v^{n-m}\right)$ (locally around any point) such that the metric $g$ takes the form

$$
g=h_{i j}(u) d u^{i} d u^{j}+\psi(u, v) k_{\mu \nu}(v) d v^{\mu} d v^{\nu}
$$

with summation over $i, j$ from 1 to $m$ and over $\mu, \nu$ from 1 to $n-m$. The condition of $g$ being nondegenerate requires $\psi$ to be non-zero and $h_{i j}$ and $k_{\mu \nu}$ to be non-degenerate everywhere; otherwise, they are arbitrary. (In the more special case that the "twisting function" $\psi$ is independent of $v$ one speaks of a warped product, cf. O'Neill [1.) It is an elementary exercise to verify that for a metric of the form (6) the submanifolds $u=$ constant are totally umbilic and the submanifolds $v=$ constant are totally geodesic. More generally, the following result is true. A semi-Riemannian manifold is (locally) a twisted product if and only if it (locally) admits two foliations $\mathcal{F}$ and $\mathcal{G}$ which are transverse and orthogonal to each other (and thus non-degenerate) with all leaves of $\mathcal{F}$ totally geodesic and all leaves of $\mathcal{G}$ totally umbilic, see Ponge and Reckziegel [8], Theorem 1. Recall that the notion of being totally umbilic is conformally invariant. So every metric that is (locally) conformal to a twisted product admits (locally) two foliations into non-degenerate totally umbilic submanifolds that are orthogonal to each other. This observation implies that a semiRiemannian manifold can be (locally) foliated into non-degenerate totally umbilic hypersurfaces if and only if it admits (locally) coordinates $(u, v)=\left(u, v^{1}, \ldots, v^{n-1}\right)$ such that

$$
g=\Phi(u, v)\left( \pm d u^{2}+\psi(u, v) k_{\mu \nu}(v) d v^{\mu} d v^{\nu}\right)
$$

Here we made use of the fact that, for a foliation into non-degenerate hypersurfaces, the orthocomplements of the leaves are one-dimensional and, thus. integrable. If we want to know if a given metric can be foliated into non-degenerate totally umbilic hypersurfaces, we may thus do this by checking whether it is isometric to (7).

\section{Totally umbilic submanifolds of Lorentzian manifolds}

From now on we assume that the ambient space $(M, g)$ has Lorentzian signature $(+, \ldots,+,-)$. We may then interpret $(M, g)$ as a spacetime in the sense of general relativity. (However, there is 
no need to restrict our discussion to the physically interesting case $\operatorname{dim}(M)=4$.) As usual in the Lorentzian case, we call the degenerate submanifolds lightlike. On a non-degenerate submanifold, the metric is either positive definite or again Lorentzian; in the first case, the submanifold is called spacelike, in the second case it is called timelike. It is our goal to discuss totally umbilic submanifolds that are timelike or lightlike. (The spacelike case is not very much different from the situation that the ambient space is Riemannian.) We begin with the timelike case.

Proposition 3. Let $\tilde{M}$ be a timelike submanifold with $2 \leq \operatorname{dim}(\tilde{M}) \leq \operatorname{dim}(M)$. Then $\tilde{M}$ is totally umbilic if and only if every lightlike geodesic that starts tangent to $\tilde{M}$ remains within $\tilde{M}$ (for some parameter interval around the starting point).

Proof. The 'only if' part is a special case of Proposition 1 (a). To prove the 'if' part, choose a point $p \in \tilde{M}$ and two vectors $X_{p}$ and $Y_{p}$ in $T_{p} \tilde{M}$ with $g_{p}\left(X_{p}, Y_{p}\right)=0$. It is our goal to prove that $\Pi_{p}\left(X_{p}, Y_{p}\right)=[0]$ because then, by the tensorial property of $\Pi$, Proposition 2 (a) proves that $\tilde{M}$ is totally umbilic. Owing to the tensorial property of $\Pi$, it suffices to consider the case that $g_{p}\left(X_{p}, X_{p}\right)=1$ and $g_{p}\left(Y_{p}, Y_{p}\right)=-1$. Then the vectors $L_{p}=\frac{1}{2}\left(Y_{p}+X_{p}\right)$ and $K_{p}=\frac{1}{2}\left(Y_{p}-X_{p}\right)$ are lightlike. By hypothesis, we can find lightlike vector fields $L$ and $K$ on $\tilde{M}$ with $\nabla_{L} L=\nabla_{K} K=0$ which take the values $L_{p}$ and $K_{p}$ at $p$. Writing $X=L-K$ and $Y=L+K$ we find $\Pi(X, Y)=\left[\nabla_{(L-K)}(L+K)\right]=\left[\nabla_{L} K-\nabla_{K} L\right]$ which is, indeed, equal to [0] because with $L$ and $K$ also the Lie bracket $[L, K]$ is tangent to $\tilde{M}$.

In general relativity, lightlike geodesics are interpreted as the wordlines of photons. Therefore, a timelike or lightlike submanifold $\tilde{M}$ is called a photon surface if each lightlike geodesic that starts tangent to $\tilde{M}$ remains within $\tilde{M}$ (for some parameter interval). In this terminology, Proposition [3] says that a timelike submanifold of dimension $k \geq 2$ is totally umbilic if and only if it is a photon surface. The notion of a photon surface was discussed by Claudel, Virbhadra, and Ellis 4 for the case $k=\operatorname{dim}(M)-1$ and by Foertsch, Hasse and Perlick [5] for the case $k=2$.

A $k$-dimensional timelike submanifold can be interpreted as the history of a $(k-1)$-dimensional spatial manifold. Proposition 3 says that this spatial manifold appears like a $(k-1)$-plane to the eye of every observer in $\tilde{M}$ if and only if its history is a totally umbilic submanifold. In particular, a 2-dimensional timelike submanifold can be interpreted as the history of a string; the condition of being totally umbilic means that the string looks like a straight line to an observer on the string. The best known non-trivial example is the surface $r=3 m, \theta=\pi / 2$ in Schwarzschild spacetime; it is the history of a circle which appears like a straight line to the eye of an observer who is situated on this circle. Other examples are worked out in [5]. There the reader can also find a characterization of 2-dimensional timelike photon surfaces in terms of inertial forces and in terms of gyroscope transport. For axisymmetric and static situations, this connection was discussed already earlier in various articles by Abramowicz, see, e.g., 7 .

If a Lorentzian manifold admits a timelike conformal Killing field $K$ that is hypersurfaceorthogonal, applying the flow of $K$ to a lightlike geodesic always gives a 2-dimensional timelike photon surface. The proof is worked out in [5]. This demonstrates the existence of 2-dimensional timelike totally umbilic submanifolds in any conformally static spacetime. Actually, for the construction to work it is not necessary that $K$ be timelike; it suffices if it is nowhere orthogonal to the lightlike geodesic to which we want to apply the flow of $K$. Most known examples of 2 -dimensional timelike photon surfaces are constructed in this way. However, this construction is not universal; with the help of Example 2 one can construct 3-dimensional Lorentzian manifolds which do not admit any non-zero comformal Killing vector field but are foliated into 2-dimensional timelike photon surfaces. 
We emphasize again that, in an $n$-dimensional Lorentzian manifold the existence of a $k$ dimensional totally umbilic timelike submanifold is not guaranteed unless in the trivial cases $k=1$ and $k=n$. This implies that, in particular, the existence of totally geodesic timelike submanifolds of dimension $2 \leq k \leq n-1$ is not guaranteed. This has physical relevance for the case $k=2$ because 2-dimensional timelike submanifolds that are totally geodesic describe the history of self-gravitating strings, see Vickers $\underline{6}$.

We now turn to the case of a lightlike submanifold $\tilde{M}$. We first observe that every such $\tilde{M}$ is ruled by a unique congruence of lightlike curves which are called the generators of $\tilde{M}$. This leads to the following characterization of totally umbilic lightlike submanifolds.

Proposition 4. A necessary condition for a lightlike submanifold to be totally umbilic is that the generators are geodesics. In a three-dimensional Lorentzian manifold, every two-dimensional lightlike submanifold $\tilde{M}$ is totally umbilic.

Proof. The first statement is a special case of Proposition 1(a). To prove the second statement, let $L$ be a vector field on $\tilde{M}$ that is tangent to the generators and $E$ any other vector field on $\tilde{M}$ that is linearly independent of $L$. Set $\nabla_{E} E=: g(E, E) N$. As $g(L, L)=0$, the vector field $\nabla_{E} L$ is perpendicular to $L$ and, thus, tangent to $\tilde{M}$. As $\nabla_{L} L$ is a multiple of $L$ and $g(E, L)=0$, the vector field $\nabla_{L} E$ is also perpendicular to $L$ and, thus, tangent to $\tilde{M}$. As a consequence, every linear combination $X=f E+h L$ satisfies $\Pi(X, X)=\left[f^{2} g(E, E) N\right]=[g(X, X) N]$. As $\Pi$ is symmetric, this proves that $\tilde{M}$ is totally umbilic.

For lightlike hypersurfaces, the generators are automatically geodesics.

Schild [12] has defined a null string as a 2-dimensional lightlike submanifold whose generators are geodesics. Whereas every 2-dimensional lightlike submanifold can be interpreted as the history of a string whose individual points move at the speed of light, null strings are characterized by the additional condition that its individual points move on geodesics, i.e., freely like photons. In other words, we may visualize a null string as a one-parameter family of photons that are arranged like perls on a string. In a three-dimensional Lorentzian manifold, every two-dimensional lightlike submanifold is totally umbilic and it is a null string in the sense of Schild.

In Schild's article it is shown that null strings can be characterized by a variational principle. From this variational principle it is clear that null strings exist in every Lorentzian manifold. This can also be verified with the help of the following construction. Choose a one-dimensional spacelike submanifold $S$; at each point of $S$, choose a lightlike direction perpendicular to $S$ that depends smoothly on the foot-point; let $\tilde{M}$ be the union of the lightlike geodesics determined by these intial directions; in a neighborhood of $S$, this is indeed a submanifold and, by construction, it is a null string. (Farther away from $S$ the set $\tilde{M}$ constructed this way may form 'caustics', i.e., it may fail to be a submanifold.)

We can try to construct $k$-dimensional totally umbilic lightlike submanifolds in Lorentzian manifolds of arbitrary dimension by the same procedure, now starting with a $(k-1)$-dimensional spacelike submanifold $S$. However, even if we are lucky enough to find a totally umbilic initial submanifold $S$, it will not be guaranteed that the resulting lightlike submanifold will be totally umbilic everywhere. We end with an example where higher-dimensional totally umbilic lightlike submanifolds can be constructed owing to the existence of symmetries.

Example 3. Consider a 4-dimensional Lorentzian manifold that is spherically symmetric and static, i.e.,

$$
g=-A(r)^{2} d t^{2}+B(r)^{2} d r^{2}+r^{2}\left(\sin ^{2} \vartheta d \varphi^{2}+d \vartheta^{2}\right),
$$


e.g. the Schwarzschild metric with $A(r)^{2}=B(r)^{-2}=1-\frac{2 m}{r}$. Let $S$ be the intersection of a hypersurface $r=$ constant with a hypersurface $t=$ constant. This is a 2-dimensional spacelike submanifold. Now choose at each point of $S$ a lightlike direction perpendicular to $S$, smoothly depending on the foot-point. (You have to choose between two possibilities: the ingoing radial directions and the outgoing radial directions.) Let $\tilde{M}$ be the union of all geodesics with the chosen initial direction. This is a submanifold near $S$. (In the case at hand, the symmetry of the situation guarantees that $\tilde{M}$ is a submanifold everywhere, except at points where it meets the center of symmetry.) It is easy to verify that $S$ is, indeed, a 3-dimensional totally umbilic lightlike submanifold. The simplest examples of totally umbilic lightlike submanifolds constructed in this way are the light cones in Minkowski space.

\section{References}

[1] B. O'Neill, Semi-Riemannian Geometry, Academic Press, New York, 1983.

[2] D. N. Kupeli, Singular semi-Riemannian geometry, Kluwer, Dordrecht, 1996.

[3] K. L. Duggal and A. Bejancu, Lightlike submanifolds of semi-Riemannian manifolds and applications, Kluwer, Dordrecht, 1996.

[4] C. M. Claudel, K. S. Virbhadra, and G. F. R. Ellis, The geometry of photon surfaces, J. Math. Phys. 42 (2001) 818-838.

[5] T. Foertsch, W. Hasse, V. Perlick, Inertial forces and photon surfaces in arbitrary spacetimes, Class. Quantum Grav. 20 (2003) 4635-4652.

[6] J. A. G. Vickers, Generalized cosmic strings, Class. Quantum Grav. 4 (1987) 1-9.

[7] M. A. Abramowicz, Centrifugal force - a few surprises, Mon. Not. R. Astr. Soc. 245 (1990) $733-746$.

[8] R. Ponge and H. Reckziegel, Twisted products in pseudo-Riemannian geometry, Geometriae Dedicata, 48 (1993) 15-25.

[9] G. Walschap, Umbilic foliations and curvature, Ill. J. Math. 41 (1997) 122-128.

[10] S. S. Ahn, D. S. Kim D-S, and Y. H. Kim, Totally umbilic Lorentzian sumbanifolds, J. Korean Math. Soc. 33 (1996) 507-512.

[11] S. K. Hong, Totally umbilic Lorentzian surfaces embedded in $L^{n}$, Bull. Korean Math. Soc. 34 (1997) 9-17.

[12] A. Schild, Classical null strings, Phys. Rev. D 16 (1977) 1722-1726. 